\title{
Bizarre Intracardiac Electrogram as an ICD Lead Damage Early Manifestation
}

\author{
Aleksander Maciąg 1,B-C,E-F, Katarzyna Gepner ${ }^{1, \mathrm{~B}-\mathrm{C}, \mathrm{F}}$, Maciej Sterliński²,A-F \\ A - Research concept and design, B - Collection and/or assembly of data, C - Data analysis and interpretation, \\ D - Writing the article, E - Critical revision of the article, F - Final approval of article
}

1 Institute of Cardiology, Warsaw

Address for correspondence:

Aleksander Maciąg, Institute of Cardiology, Warsaw email: amaciag@ikard.pl

Katarzyna Gepner, Institute of Cardiology, Warsaw email: kasia.gepner@wp.pl

Maciej Sterliński, Institute of Cardiology, Warsaw email: msterlinski@poczta.onet.pl

\begin{abstract}
Implantable cardioverter-defibrillator (ICD) therapy is associated with an increasing number of ICD lead-related complications. We present a case of 52-year-old female treated with ICD for the post-infarction primary prophylaxis in whom inappropriate shocks were caused by lead damage manifested early by prodromal, unusual intracardiac electrogram (IEGM) artifacts. Some IEGM patterns should be considered
\end{abstract}

as a potential marker of lead-related adverse events that may occur in the future

\section{ICD, lead damage, IEGM manifestation}

Implanting a cardioverter-defibrillator (ICD) is a commonly accepted method to improve survival in post-myocardial infarction (MI) patients with left ventricle dysfunction ${ }^{1}$. The benefit of this therapy is downgraded by a considerable number of disadvantages ${ }^{2}$. They include an increasing number of ICD lead-related complications. Inappropriate interventions caused by sensing bias are often the first sign of lead damage; they may proceed with further complete lead continuity break, resulting in loss of pacing or defibrillation. Routine ICD patients' follow-up with an intracardiac electrogram (IEGM) and electrical parameters interrogation helps to disclose the problem and provide adequate management ${ }^{3}$.

We present the case of a 52-year-old woman who received five years earlier a single chamber ICD for primary prophylaxis due to post-MI left ventricle ejection fraction 30\% and non-sustained ventricular tachycardia (VT). Within six months after the implantation, a successful ATP intervention occurred for VT and two inadequate shocks in the ventricular fibrillation zone provoked by sinus tachycardia (after withdrawal of B-blockers). The medication was restored but an additional V-ring - can IEGM channel was set to broaden the spectrum of diagnostics. No interventions were noted for the next two years, then eight shocks within one month were delivered. Interrogation (three months after the previous follow-up) showed an increase of pacing threshold from $0.75 \mathrm{~V}$ to 1.5 $\mathrm{V}$ and a pacing resistance decrease from 305 to $175 \mathrm{ohms}$. The R-wave remained stable at $>12 \mathrm{mV}$. High voltage circuit (HV) resistance was in the normal range. The device memory revealed shocks caused by an unusual and bizarre finding in IEGMs (shown in figure 1, strip A). A final proof of the damage was the last ICD discharge IEGM with typical damage bias in the V-tip - V-ring channel (shown in figure 1, strip B). The patient was qualified for implantation of a new lead. Since the re-intervention the clinical course of the therapy has remained uncomplicated. 

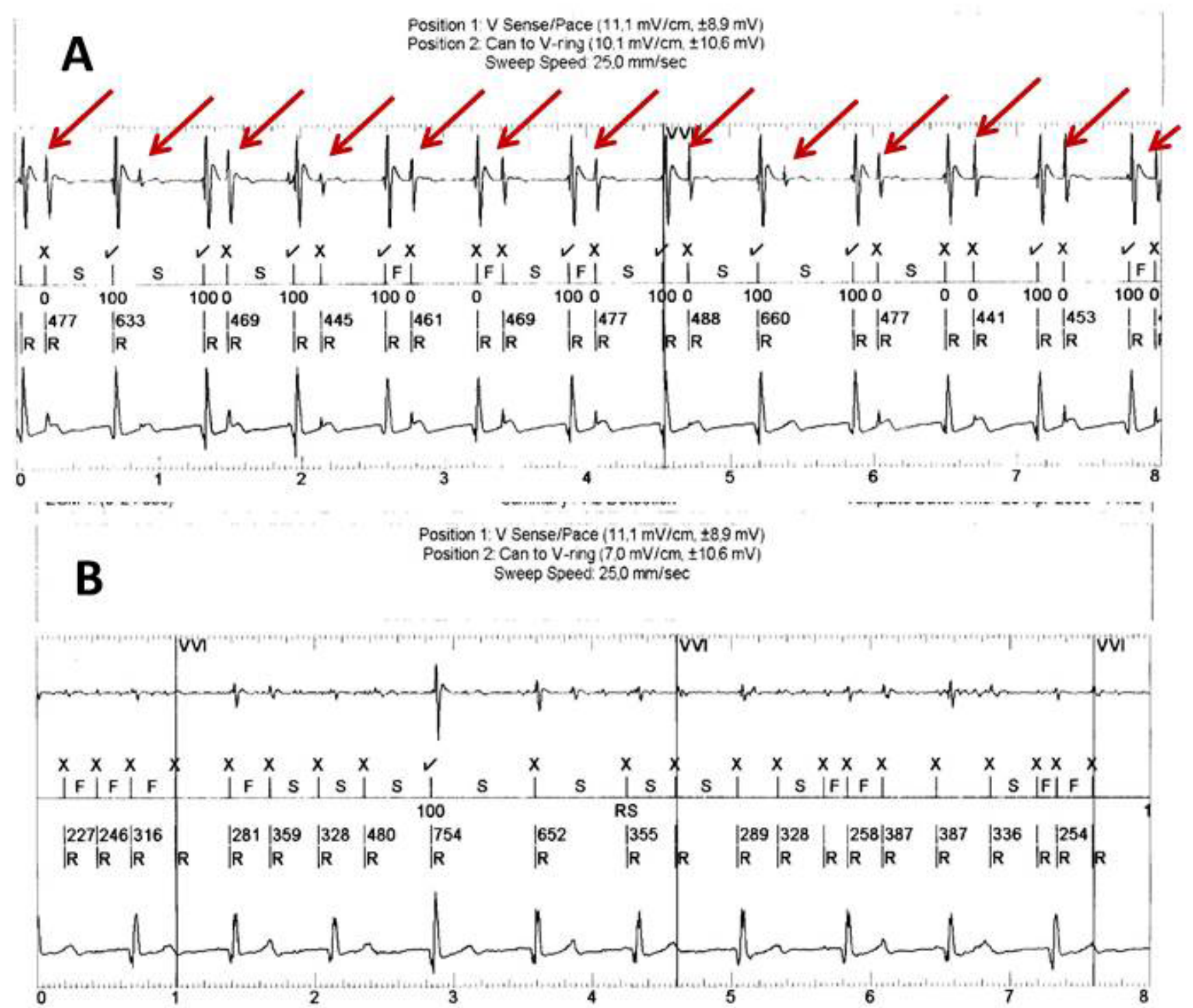

Figure 1 A. Unusual lead damage IEGM manifestation. Red arrows indicate regular artifacts generated assumably by systolic heart movement. Artifacts can be seen in $\mathrm{V}$ sense and in can to $\mathrm{V}$-ring channel as well what suggests $\mathrm{V}$ ring circuit damage involvement. B. Common lead damage IEGM manifestation later, at the same patient. V - ventricular, IEGM - intracardiac electrogram.

As the ICD population broadens and expected patient survival increases, proper monitoring, troubleshooting and identifying potential therapy complications is a growing problem. In this case collected data suggested damage of the outer V-ring conductor and probably - due to low pacing resistance - of the inner insulation in the distal lead part. Artifacts were present not only in the V-tip - V-ring but also in the surface-like V-ring - can channel as well (shown in figure 1, strip A). Spikes were absent in classic 12-lead surface electrograms. The regular and repeatable coupling interval between QRS and artifacts might indicate heart systolic movement to generate false spikes counted as an arrhythmic event, when falling in the VT or VF frequency window. A similar IEGM pattern may sometimes suggest and mimic lead dislocation to the coronary sinus region, but parallel V-ring - can IEGM registration and stable lead location in the right ventricle, seen in chest X-ray, easily exclude this possibility. Normal $\mathrm{HV}$ resistance indicated no defibrillation circuit involvement, but as the lead damage was diagnosed, implantation of a new lead was necessary anyway.
In conclusion, all ICD patients should be followed carefully and all the interrogated data should be analyzed, especially when inappropriate therapies occur ${ }^{3}$. Telemonitoring features to supervise lead performance should be emphasized ${ }^{5}$. Unnecessary shocks can be avoided by reprogramming but due to an increasing number of failing ICD leads that may range from $2 \%$ to even $20 \%$ a year ${ }^{4}$, a proper diagnosis may indicate re-intervention as the only treatment.

The presented uncommon early lead damage manifestation may sometimes be unclear and make the early curative decision difficult.

\section{References}

1. Priori SG, Blomström-Lundqvist C, Mazzanti A et al. 2015 ESC Guidelines for the management of patients with ventricular arrhythmias and the prevention of sudden cardiac death: The Task Force for the Management of Patients with Ventricular Arrhythmias and the Prevention of Sudden Cardiac Death of the European Society of Cardiology (ESC). Endorsed by: Association 
for European Paediatric and Congenital Cardiology (AEPC). Eur Heart J. 2015 Nov 1;36(41):2793-867.

2. Gepner K, Przybylski A, Maciąg A et al. Causes of redo procedures in patients with an implantable cardioverter-defibrillator - long-term follow-up results Kardiol Pol 2007; 65: 893-898

3. Daubert JP, Zareba W, Cannom DS et al. Inappropriate implantable cardioverter-defibrillator shocks in MADIT II: frequency, mechanisms, predictors, and survival impact. J Am Coll Cardiol. 2008;51(14):1357-65.

4. Kleemann T, Becker T, Doenges K et al. .Annual Rate of Transvenous Defibrillation Lead Defects in Implantable Cardioverter-Defibrillators Over a Period of $>10$ Years. Circulation. 2007;115:2474-2480.

5. Hindricks G, Taborsky M, Glikson M et al. ; IN-TIME study group. Implant-based multiparameter telemonitoring of patients with heart failure (IN-TIME): a randomised controlled trial. Lancet. 2014 Aug 16;384(9943):583-90. 